Document downloaded from:

http://hdl.handle.net/10251/144682

This paper must be cited as:

Llopis-Lorente, A.; Diez-Sánchez, P.; Sánchez, A.; Marcos Martínez, MD.; Sancenón Galarza, F.; Martinez-Ruiz, P.; Villalonga, R.... (02-2). Toward chemical communication between nanodevices. Nano Today. 18:8-11. https://doi.org/10.1016/j.nantod.2017.09.003

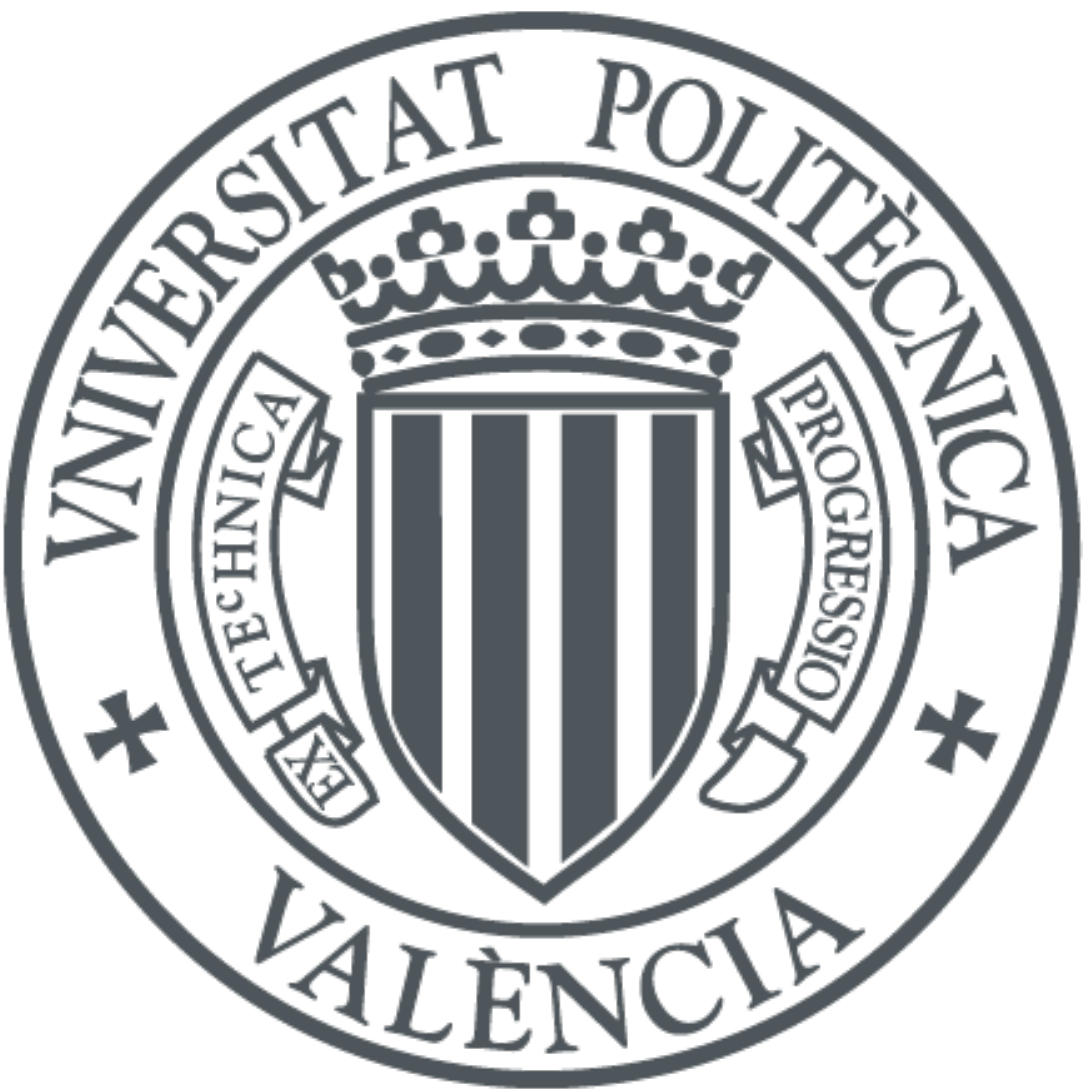

The final publication is available at

https://doi.org/10.1016/j.nantod.2017.09.003

Copyright Elsevier

Additional Information 


\section{Toward chemical communication between nanodevices}

Antoni Llopis-Lorente, ,a,b,c] Paula Díez, ${ }^{[d]}$ Alfredo Sánchez, ${ }^{[d, e]}$ María D. Marcos, ${ }^{[a, b, c]}$ Félix Sancenón, ${ }^{[a, b, c]}$ Paloma Martínez-Ruiz, ${ }^{[e]}$ Reynaldo Villalonga ${ }^{[d] *}$ and Ramón Martínez-Máñez ${ }^{[a, b, c] *}$

ABSTRACT: Although many nanodevices have been described in recent years, nanoparticles capable of communicating with each other have been barely reported. Traditional communication technologies cannot be applied on the nanoscale, but a potential approach to achieve this goal is to mimic how nature communicates by exchanging information using molecules. Based on these concepts, some examples using DNA, enzymes and small molecules for information processing, nanoparticles capable of modulating chemical communication between cells and nanoparticles that can communicate with each another have been reported. Communication between nanodevices may find applications in different areas and a number of future new results are envisioned in this research field.

Ever since Richard Feynman gave his famous lecture "There's plenty of Room at the Bottom" in 1959 [1], significant advances in the nanotechnology field have been made. Nanotechnology is a highly multidisciplinary field with a steadily growing number of applications in different areas, such as medicine [2] electronics [3], chemistry [4] and computing [5]. Nanotechnologists work on developing devices that range from one to a few hundred nanometers capable of performing certain tasks, with examples such as nanomemories [6], nanobatteries [7], nanocarriers [8] and nanomotors [9] already having been developed. To date, these nanomachines have been studied mainly as single isolated units, and relatively very few examples are available in which human-made nanoconstructs communicate with one another. However, this is an appealing research field, and communication or interconnection between nanosystems is expected to lead to a number of new and advanced functionalities [10].

\footnotetext{
[a] Instituto Interuniversitario de Investigación de Reconocimiento Molecular y Desarrollo Tecnológico (IDM), Unidad Mixta Universidad Politécnica de Valencia-Universidad de Valencia (Spain)

[b] Departamento de Química, Universidad Politécnica de Valencia Camino de Vera s/n, 46022, Valencia (Spain)

E-mail: rmaez@qim.upv.es

[c] CIBER de Bioingeniería, Biomateriales y Nanomedicina (CIBER-BBN) (Spain)

[d] Department of Analytical Chemistry, Faculty of Chemistry, Complutense University of Madrid, 28040 Madrid (Spain) E-mail: rvillalonga@quim.ucm.es

[e] Department of Organic Chemistry I, Faculty of Chemistry, Complutense University of Madrid, Madrid 28040, Spain.
} 
Communication has become essential to human technology, but has barely been studied on the nanoscale. However, traditional communication technologies cannot be applied nanometrically as transmitters and receivers are still too big to fit on a nanoparticle. In line with this, the development of technologies to communicate nanodevices requires new ideas and concepts that can be applied on the nanoscale. Such technologies should employ components that can be easily incorporated into nanoparticles and to provide communication with minimum energy use. In this scenario, a potential approach to establish communication at the nanometric level is to mimic how nature communicates. In fact, a number of biological processes are governed by communication between cells. This form of communication, also called molecular communication, consists in information exchange using molecules. Thus neurons propagate information by means of neurotransmitter molecules [11]. For instance, acetylcholine is released from synaptic vesicles and is recognized by cholinergic receptors on muscle cells, which triggers muscle contraction. In the pancreas, $\beta$-cells respond to high levels of glucose and secret insulin, which travels through the blood to receptors on distant cells [12]. Activation of enzymes and vital processes, such as digestion, respiration and sleep, are regulated by hormones and other molecules that provide communication between organs [13]. Pheromones are used by bacteria and insects like ants to communicate with members of their community [14]. Unlike the systems used in telecommunication or computer engineering, it is feasible to integrate molecular communication into nanodevices and their power consumption is expected to be very low compared to traditional telecommunication technologies. However, there are still many open questions that need to be solved in the future; for example, which molecules are to be used to encode information?; how can these molecules be recognized by different nano-objects?; how is this recognition transformed into information propagation? Answering these issues is no trivial task.

In communication theory terms, communication can be defined as the process of establishing a connection between two points. At one point of the communication process, there is the sender, which/who receives a stimulus to send a message across. The sender transforms information into a code and transmits the message through a suitable medium. The receiver perceives the message and decodes it. Communication is considered effective if it finally results in a desired result, response or reaction.

Some groups have already reported stimulating advances by using biomolecules for information processing with the potential of being incorporated into inter-nanodevice communication. As in digital circuits, where signals are processed with AND, OR and NOT logic gates, Winfree and co-workers constructed logic gates using different DNA strands as inputs 
and outputs [15]. Based on a mechanism of sequence recognition and strand displacement, one strand (or more) was able to recognize input strands and to release other output strands. Moreover, the output strand could serve as input for another logic gate or could produce an output fluorescent signal after displacement. Following these principles, complex networks have been constructed using DNA strand displacement cascades [16]. Enzymes have also been employed to mimic logic gates and enzymatic communication cascades [17]. Such ensembles rely on specific enzyme-substrate recognition, the generation of new products that can serve as inputs for other enzymes in the network, and the regulatory effects of cofactors and inhibitors. For instance, Katz et al. reported a network of three concatenated AND logic gates based on three enzymes (invertase, glucose oxidase and catalase) and three enzymatic reactions, which finally resulted in the formation of a colored product as an output signal [18]. Willner and co-workers constructed a hybrid system in which DNA scaffolds served to assemble enzymatic cascades [19]. Synthetic chemical systems [20] and gold nanoparticles [21] capable of transforming a combination of chemical signals into fluorescence and color changes have also been reported.

Interestingly, researchers have also recently started to explore how to program and modulate chemical communication between cells. Basu et al. reported the use of genetically modified 'sender' cells that secreted the chemical acyl-homoserine lactone, which induced 'receiver' cells to form ring-like patterns of differentiation [22]. Payne and co-workers reported having prepared gelatin-alginate beads to entrap 'sender cells', which synthesized and secreted a specific chemical that was taken up by $\mathrm{E}$. Coli, inducing the synthesis of a fluorescent protein [23]. Others authors have reported using phospholipid vesicles that contained a DNA template, transcription-translation machinery and isopropyl $\beta$-D-1thiogalactopyranoside (IPTG) to transform the presence of certain chemicals in communication with bacteria. In the presence of theophylline, the synthesis of a pore-forming protein in vesicles was activated, which led to the release of IPTG. In turn, this IPTG was detected by E. Coli that produced a fluorescent protein and closed the transduction loop [24]. Recently, the same group reported engineered vesicles capable of mediating communication between two different strains of bacteria. Signaling molecules secreted by the first bacterium induced the synthesis and release of a chemical from the vesicles, which induced the production of green fluorescent protein in the second bacterium [25].

However, communication between abiotic nanoparticles has practically not been explored. In this field, Martínez-Máñez and co-workers proposed a communicating system based on three different gated mesoporous silica nanoparticles that exchanged chemical 
messengers [26]. A communication cascade was triggered by the enzyme pancreatin, which hydrolyzed the oligosaccharide cap of a first nanoparticle (NP-A) to result in the delivery of reductor (tris(2-carboxyethyl)phosphine, TCEP). TCEP cleaved a disulfide bridge uncapping a second nanoparticle (NP-B) and inducing the delivery of a surfactant (dodecyltrimethylammonium bromide), which finally disrupted the lipid bilayer of a third nanoparticle (NP-C) which, in turn, resulted in the delivery of a dye (safranin O). In this communication system, delivery from the third NP-C nanoparticle is governed by an input into NP-A and by a cascade communication between nanoparticles using two chemical messengers. In communication theory terms, this is a linear model of communication, and the message flows in a straight line from the sender (NP-A) to the receiver (NP-C) by using intermediate nanoparticles (NP-B) that works as both the receiver and sender.

More recently, we reported a communicating system based on enzyme-functionalized Janus gold-gated mesoporous silica nanoparticles that mimics an interactive model of communication in which the sender sends a message to the receiver, and the receiver then channels a message back to the original sender. [27] Janus nanoparticles consisted of mesoporous silica nanoparticles covalently anchored to a gold nanoparticle. In this case, a first nanoparticle (JNP-A) was functionalized with the enzyme $\beta$-galactosidase on the gold nanoparticle and the mesoporous face was loaded with a dye and capped with $\beta$-cyclodextrin $(\beta-C D)$ attached to the silica surface through disulfide bonds. In a second nanoparticle (JNP-B) the mesoporous part was loaded with a reducing agent ( $\mathrm{N}$-acetyl-L-cysteine) and capped with a $\mathrm{pH}$-responsive supramolecular nanovalve, whereas the gold side was functionalized with glucose oxidase. The communication process was triggered upon the addition of lactose. Lactose was hydrolyzed by $\beta$-galactosidase on JNP-A and the generated glucose (the first chemical messenger) diffused to JNP-B, where glucose oxidase transformed glucose into gluconic acid. This results in lowering the local $\mathrm{pH}$, which produced the uncapping of the supramolecular nanovalve delivering the entrapped $\mathrm{N}$-acetyl-L-cysteine (the second chemical messenger), which diffused as feedback toward JNP-A. N-acetyl-L-cysteine, induced the rupture of the disulfide linkages on the mesoporous face of JNP-B and the release of the entrapped dye. Cargo delivery from the first nanoparticle was observed only after biunivocal communication with the second nanoparticle. In this case communication was mediated by two enzymatic processes and the exchange of two chemical messengers. These examples rely on the use of mesoporous silica, which has interesting properties, such as a high loading capacity, chemical stability and the possibility of functionalization with molecular gates that control the release of a cargo when an external stimulus is applied. The gold part in the Janus 
particles allows a single nanodevice to have two different surfaces. Notwithstanding, we believe that the properties of other materials, such as metallic or polymeric nanoparticles, could be used to develop different communication protocols.

Communication between nanodevices will allow nanoparticles to cooperate, share information and perform advanced tasks. New communication paradigms are expected to be developed in the near future and applications in a number of different areas are also expected. For instance, the design of nanoparticles capable of working cooperatively to enhance the delivery of drugs is envisioned. In this scenario, Bathia and coworkers have already provided an example of how tumor-targeted gold nanorods triggered a coagulation cascade, which increased the uptake of drug-loaded liposomes by the tumor and, therefore, enhanced treatment efficiency [28]. From another point of view, different nanoparticles can act as nanocontainers capable of delivering a certain cargo on-command and of inducing the in situ synthesis of certain compounds of interest. In this field, Willner and co-workers have shown that certain chemicals loaded in mesoporous silica nanoparticles can be accurately delivered when selected stimuli are applied, and react between them to yield new molecules [29]. Another potential application could be the detection of low levels of target analytes by amplifying the signal through a cascade communication process with other nanoparticles. More advanced cooperative systems can also be envisioned in these fields (nanomedicine, in situ synthesis, sensing and others), in which circulating sensing nanoparticles collect information and transmit its information to static nanodevices that could emit an output signal or communicate to other actuating nanomachines. Although there is still a long way to go until these futuristic applications become a reality, and the described communication systems on the nanoscale are still far from complex, the design of new communication paradigms is envisioned to have a great impact in the chemical community.

In communication terms, on the nanoscale new advances in the knowledge of how the recognition of individual molecules (via chemical or biochemical reactions) can be used to encode information, and how this information can propagate, will require the collaboration of researchers from different fields. Moreover, the concept of creating communication between nanodevices embraces an enormous potential to prepare new and more advanced cooperative nanoscale systems whose response is governed by communication processes between individual nanoparticles. Inspired by how biological and humans communicate, the development of such systems will open up new directions in different areas and a number of new advances are envisioned in the near future. 


\section{Acknowledgements}

A. Llopis-Lorente is thankful to the "La Caixa" Banking Foundation for his PhD grant. The authors are grateful to the Spanish Government (MINECO Projects MAT2015-64139-C4-1, CTQ2014-58989-P, CTQ2015-71936-REDT and AGL2015-70235-C2-2-R) and the Generalitat Valenciana (Project PROMETEOII/2014/047). The authors also thank the Comunidad de Madrid (S2013/MIT-3029, Programme NANOAVANSENS) for support.

\section{References}

[1] R. P. Feynman, Caltech Eng. Sci. 23 (1960) 22-36.

[2] J. Shi, A. R. Votruba, O. C. Farokhzad, R. Langer, Nano Letters 10 (2010), 3223-3230.

[3] W. Lu, C. M. Lieber, Nat. Mater. 6 (2007) 841-850.

[4] E. C. Dreaden, A. M. Alkilany, X. Huang, C. J. Murphy, M. A. El-Sayed, Chem. Soc. Rev. 41 (2012) 2740-277.

[5] D. S. Markovic, D. Zivkovic, D. Cvetkovic, R. Popovic, Renew. Sust. Energ. Rev. 16 (2012) 29662972.

[6] R. Tseng, J. Huang, J. Ouyang, R. Kaner, Y. Yang, Nano Lett. 5 (2005) 1077-1080.

[7] I. Valov, E. Linn, S. Tappertzhofen, S. Schmelzer, J. van den Hurk, F. Lentz, R. Waser, Nat. Commun. 4 (2013). DOI: 10.1038/ncomms2784.

[8] E. Aznar, M. Oroval, L. Pascual, J. R. Murguía, R. Martínez-Máñez, F. Sancenón, Chem. Rev. 116 (2016) 561-718.

[9] T. Kline, W. Paxton, Angew. Chem. Int. Ed. 44 (2005) 744-746.

[10] I. F. Akyildiz, F. Brunetti, C. Blázquez, Comput. Networks 52 (2008) 2260-2279.

[11] K. M. Betke, C. A. Wells, H. E. Hamm, Prog. Neurobiol. 96 (2012) 304-321.

[12] A. R. Saltiel, C. R. Kahn, Nature 414 (2001) 799-806.

[13] K. G. Murphy, S. R. Bloom, Nature 444 (2006) 854-859.

[14] R. Wirth, A. Muscholl, G. Wanner, G. Trends Microbiol. 4 (1996) 96-103.

[15] G. Seelig, D. Soleveichik, D. Y. Zhang, E. Winfree, Science 314 (2006) 1585-1590.

[16] L. Qian, E. Winfree, J. Bruck, Nature 475 (2011) 368-372.

[17] E. Katz, V. Privman, Chem. Soc. Rev. 39 (2010) 1835-1837.

[18] G. Strack, M. Ornatska, M. Pita, E. Katz, J. Am. Chem. Soc. 130 (2008) 4234-4235.

[19] O. I. Wilner, Y. Weizmann, R. Gill, O. Lioubashevsky, R. Freeman, I. Willner, Nat. Nanotech. 4 (2009) 249-254.

[20] A. P. de Silva, N. D. McClenaghan, Chem. Eur. J. 10 (2004) 574-586.

[21] D. Liu, W. Chen, K. Sun, K. Deng, W. Zhang, Z. Wang, X. Jiang, Angew. Chem. Int. Ed. 50, (2011) 4103-4107.

[22] S. Basu, Y. Gerchman, C. H. Collins, F. H. Arnold, R. Weiss, Nature 434 (2005) 1130-1135.

[23] Y. Liu, H.-S. Wu, M. Chhuan, J. L. Terrel, C.-Y. Tsao, W. E. Bentley, G. F. Payne, ACS Biomater. Sci. Eng. 1 (2015) 320-328.

[24] R. Lentini, S. Pérez Santero, F. Chizzolini, D. Cecchi, J. Fontana, M, Marchioretto, C. Del Bianco, J. L. Terrel, A. C. Spencer, L. Martini, M. Forlin, M. Assafalg, M. Dalla Serra, W. E. Bentley, Nat. Commun. 5 (2014). DOI: 10.1038/ncomms5012.

[25] R. Lentini, N. Y. Martín M. Forlin, L. Belmonte, J. Fontana, M. Cornella, L. Martini, S. Tamburini, W. E. Bentley, O. Jousson, S. S. Mansy, ACS Cent. Sci. (2017) 117-123.

[26] C. Giménez, E. Climent, E. Aznar, R. Martínez-Máñez, F. Sancenón, M. D. Marcos, P. Amorós, K. Rurack, Angew. Chem. Int. Ed. 53 (2014) 12629-12633.

[27] A. Llopis-Lorente, P. Díez, A. Sánchez, M. D. Marcos, F. Sancenón, P. Martínez-Ruíz, R. Villalonga, R. Martínez-Mañez, Nat. Commun. 8 (2017). DOI: 10.1038/ncomms15511. 
[28] G. von Maltzahn, J.-H. Park, K. Y. Lin, N. Singh, C. Schwöppe, R. Mesters, W. E. Berdel, E. Ruoslahti, M. J. Sailor, S. N. Bhatia, Nat. Mat. 10 (2011) 545-552.

[29] D. Balogh, M. A. Alemán-García, H. Bauke-Albada, I. Willner, Angew. Chem. Int. Ed. 54 (2015) 11652-11656.

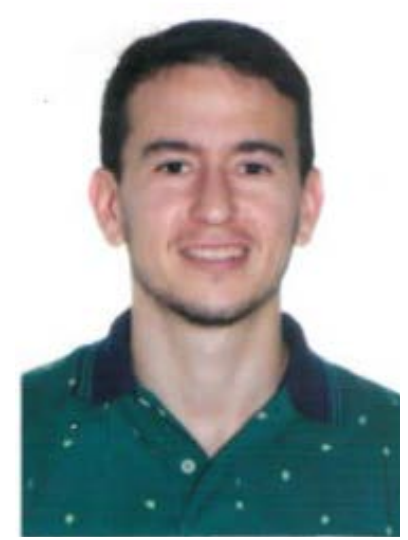

Antoni Llopis-Lorente studied Chemistry at the University of Valencia and graduated with honors in 2013. Currently, he is a PhD candidate at the Polytechnic University of Valencia (UPV) under the supervision of Prof. Ramón Martínez-Mañez. He has carried out research stays at the Complutense University of Madrid (Spain) and the Radboud University (the Netherlands) in the group of Prof. Reynaldo Villalonga and the group of Prof. Daniela Wilson, respectively. His main research interests are to develop enzyme-based nanodevices and nanoparticles for sensing, drug delivery and chemical communication.

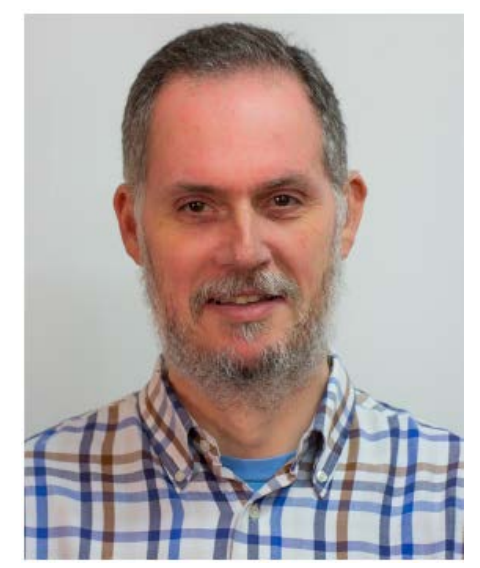

Félix Sancenón was born in 1968 in Manises, Valencia (Spain), and graduated in Chemistry in 1991. He received his PhD degree in 2003. Afterward, he worked with Professor L. Fabbrizzi at the Universitá di Pavia on the synthesis of chromogenic receptors for ion pairs. Then he joined the Department of Chemistry at the Polytechnic University of Valencia with a Ramón y Cajal contract. He became a lecturer in 2006. He is the coauthor of more than 220 research publications. His current research interest comprises the use of hybrid materials to develop sensors and to construct molecular gates. 


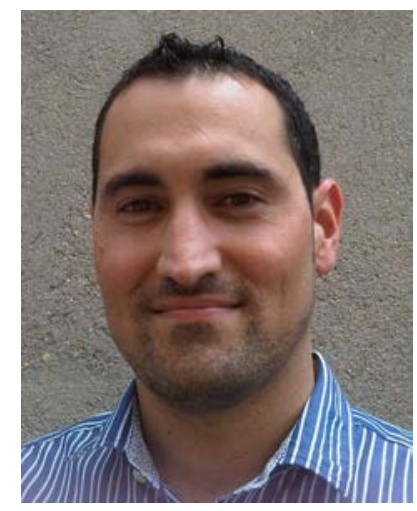

Alfredo Sánchez was born in 1983 in Madrid, Spain. He received his B.S. degree in Chemical Engineering from the Rey Juan Carlos University in 2006 and he obtained his PhD degree at the same university in 2011. Since 2012 he has worked at the Complutense University of Madrid under the supervision of Prof. Reynaldo Villalonga and Prof. José M. Pingarrón in the design of enzyme biosensors and enzymatic-based hybrid nanomaterials.

María Dolores Marcos (right) was born in Valencia, Spain, and received her PhD in chemistry from the University of Valencia in 1992 and was a postdoctoral fellow at Cambridge University, UK. She has been a lecturer in the Department of Chemistry at the Polytechnic University of Valencia since 2000. At present she undertakes her research at the IDM Research Institute of the Polytechnic University of Valencia. She is the coauthor of more than 120 research publications and ten patents. Her current research interest involves designing hybrid materials to develop new methods for sensing and for controlled delivery applications. 


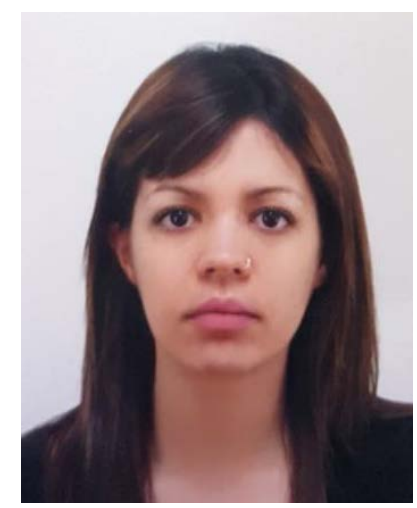

Paula Díez was born in 1986 in León, Spain, and received her B.S. degree in Chemistry from the Complutense University of Madrid in 2011. She obtained her PhD degree at the same university in 2016 where she is currently working under the supervision of Prof. Reynaldo Villalonga. She has been working as a visitor researcher at the University of California (San Diego) in Prof. Joseph Wang's group, and also at the Polytechnic University of Valencia (UPV) with Prof. Ramón Martínez-Mañez's group. Her research focuses on enzyme biosensors and smart nanomaterials for drug delivery and chemical communication.

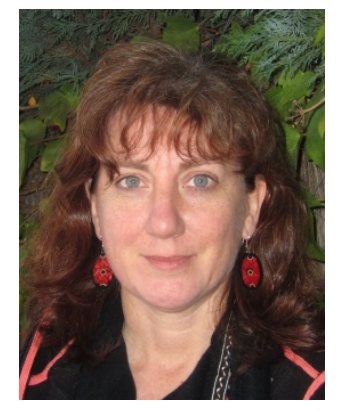

Paloma Martínez Ruiz obtained her PhD degree in Organic Chemistry in 1997 from the Complutense University of Madrid. She received predoctoral (1996) and postdoctoral (1998) training at the Organic Chemistry Institute of Eberhard-Karls Univertät Tübingen (Germany) under the supervision of Prof. Dr. h.c. Michael Hanack. She has been an assistant professor of Organic Chemistry at the Complutense University of Madrid since 2009. She has collaborated in more than 15 national and international research projects, and has been the PI of two projects. In 2012, she started collaborating with Dr. Villalonga on the synthesis of novel nanostructured materials to develop bioactive nanodevices and biosensors.




Reynaldo Villalonga was born in 1970 in Matanzas, Cuba. He obtained his BSc and PhD degree in Chemistry at the University of Havana in 1993 and 2001, respectively. In 1996 he moved to the University of Matanzas, Cuba, where he founded the Enzyme Technology Group. At this university he was appointed as Full Professor and the Founding Director of the Center for Enzyme Technology in 2007. In 2010 he moved to the Complutense University of Madrid, Spain, where he held a Ramón y Cajal Research Professorship and was further appointed as an assistant professor of Analytical Chemistry and the head of the Nanosensors and Nanomachines Research Group. His research focuses on nanomaterials engineering, biosensors, neoglycoenzymes, carbohydrate chemistry and drug delivery systems. He has been recognized with several honors and awards, including seven prizes from the Cuban Academy of Sciences, the Development Cooperation Prize of Belgium (2001), the Third World Academy of Sciences Award for Young Chemists (2004), the National Award in Chemical Sciences (2006), the Prize of the Cuban Ministry of Science (2007), the Prize of the Ministry of Higher Education (2004, 2007), the Japanese Society for the Promotion of Science Fellowship $(2004,2008)$, and the Carolina Foundation Fellowship (2007).

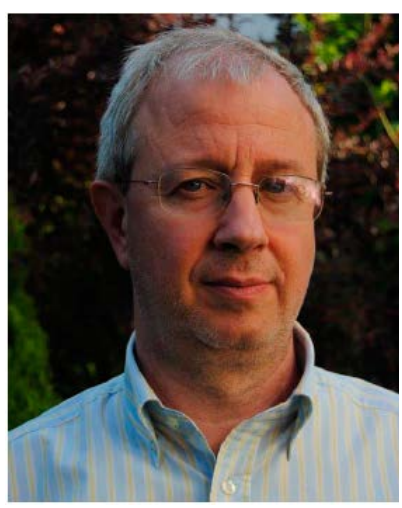

Ramón Martínez-Máñez is a full Professor of Inorganic Chemistry in the Department of Chemistry at the Polytechnic University of Valencia (UPV), is the Head of the Interuniversity Research Institute for Molecular Recognition and Technological Development (IDM) in Valencia and the Scientific Director of the Biomedical Research Center Network in Bioengineering, Biomaterials and Nanomedicine (CIBER BBN). He has more than 380 publications ( $h$-index 61 ) of which more than 100 have been published in leading general chemistry journals. He has coauthored one scientific reference book published by Wiley in 2010 and is the co-author of 15 book chapters. He holds supervised $33 \mathrm{PhD}$ theses and more than 20 patents. He is the CoChairman of the journal ChemistryOpen and a member of the International Advisory Board of the journals Chem. Asian. J. and ChemPlusChem, all published by Wiley-VCH. Recently he received the Research Excellence Award 2016 from the Real Sociedad Española de Química. He has been an active researcher in the fields of chromo-fluorogenic probes to detect ions and molecules of biological and environmental importance, some of which are based on hybrid nanomaterials. Moreover, he has also been working on the design of innovative gated nanodevices for controlled drug release using external stimuli. 